Received: 29.11 .2019

Revised: 28.01 .2020

Accepted: 21.02 .2020

DOI: $10.17804 / 2410-9908.2020 .1 .024-033$

\title{
EFFECT OF HYDROGENATION TEMPERATURE AND TENSILE STRESS ON THE PARAMETERS OF THE COMPLETE DEFORMATION DIAGRAM FOR STEEL 09G2S
}

\author{
V. I. Mironov ${ }^{1,2, a)}$, I. G. Emelyanov ${ }^{1, \text { b) }}$, D. I. Vichuzhanin ${ }^{1, c)}$, L. M. Zamaraev ${ }^{1, d)}$, \\ D. A. Ogorelkov ${ }^{1,2, \text { e)* }}$, and V. V. Yakovlev ${ }^{2, \text { f) }}$ \\ ${ }^{I}$ Institute of Engineering Science, Ural Branch of the Russian Academy of Sciences, \\ 34 Komsomolskaya St., Ekaterinburg, 620049, Russian Federation \\ ${ }^{2}$ B. N. Yeltsin Ural Federal University, \\ 19 Mira St, Ekaterinburg, 620002, Russian Federation

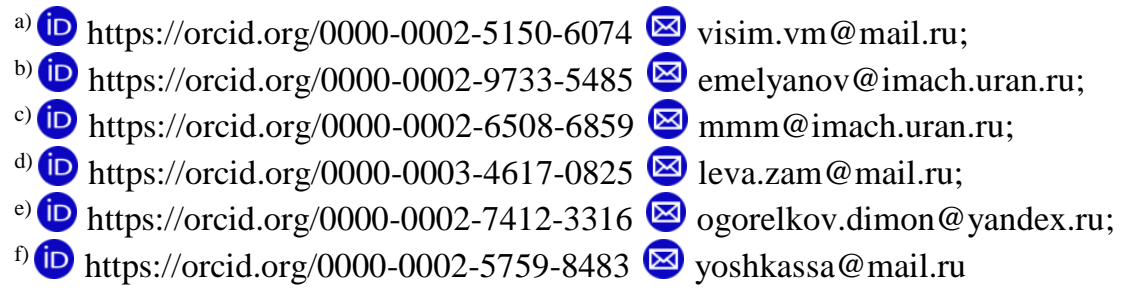

\footnotetext{
*Corresponding author. E-mail: visim.vm@mail.ru, mailto: visim.vm@mail.ru Address for correspondence: 34 Komsomolskaya St., Ekaterinburg, 620049, Russian Federation Tel.: +79221558983
}

Hydrogen concentration in the sample material depends on its environmental content, external pressure, temperature, surface condition, and testing time. All other things being equal, increasing temperature increases the diffusion coefficient and, consequently, hydrogen concentration in the metal. This, in turn, changes the mechanical properties of the material. The paper considers the principal possibility of determining the parameters of this process within the method of complete deformation diagrams. By changing the parameters of the diagram of the hydrogenated metal, a conclusion is made about the changes in its strength, plasticity, and crack resistance. The approach is illustrated by the results of testing samples of the structural $09 \Gamma 2 \mathrm{C}$ steel, widely used in mechanical engineering. It has been found that the increasing temperature of hydrogenation reduces the strength and ductility of the steel and increases crack resistance. Thus, it is experimentally proved that the parameters of the falling branch of the complete diagram are sensitive to hydrogen concentration in the steel. The changes in the mechanical properties of the structural steel caused by the action of hydrogen are qualitatively similar to those caused by cyclic loading. In this regard, it is concluded that degradation processes of different natures can be studied on a common methodological basis. The parameters of the falling branch of the complete deformation diagram can serve as the representative parameters of these processes.

Keywords: hydrogen, diffusion, experiment, complete deformation diagrams, temperature.

\section{References}

1. Bolotin V.V. Resurs mashin i konstruktsiy [Resource of Machines and Structures]. Moscow, Mashinostroenie Publ., 1990, 448 p. (In Russian).

2. Plyutov Yu.A., Homich L.V. New approaches to analyzing the reliability of vehicles. Fundamentalnye Issledovaniya, 2004, no. 6, pp. 70-72. (In Russian). 
3. Emel'yanov I.G, Mironov V.I. Dolgovechnost obolochechnykh konstruktsiy [Durability of Shell Structures]. Ekaterinburg, RIO UrO RAN Publ., 2012, 217p. ISBN 978-5-7691-2322-1. (In Russian).

4. Yakovleva T.Yu. Lokalnaya plasticheskaya deformatsiya i ustalost metalla [Local Plastic Deformation and Fatigue of Metals]. Kiev, Naukova Dumka Publ., 2003, 238 p. (In Russian).

5. Moroz L.S., Chechulin B.B. Vodorodnaya khrupkost metallov [Hydrogen Brittleness of Metals]. Moscow, Metallurgy Publ., 1967, 256 p. (In Russian).

6. Suzuki H., Fukushima H., Takai K. Role of Hydrides and Solute Hydrogen in Embrittlement of Pure Titanium. Journal of the Japan Institute of Metals, 2015, vol. 79, pp. 82-88. DOI: 10.2320/jinstmet.JC201402.

7. Tal-Gutelmacher E., Eliezer D. Hydrogen-Assisted Degradation of Titanium Based Alloys. Materials Transactions, 2004, vol. 45, iss. 5, pp. 1594-1600. DOI: 10.2320/matertrans.45.1594.

8. Venezuela J., Blanch J., Zulkiply A., Liu Q., Zhou Q., Zhang M., Atrens A. Further study of the hydrogen embrittlement of martensitic advanced high-strength steel in simulated auto service conditions. Corrosion Science, 2018, vol. 135, pp. 120-135. DOI: 10.1016/j.corsci.2018.02.037.

9. Garetta G., Cioffi P., Bruschi R. Engineering thoughts on Hydrogen Embrittlement. Procedia Structural Integrity, 2018, vol. 9, pp. 250-256. DOI: 10.1016/j.prostr.2018.06.038.

10. Lynch S. Hydrogen embrittlement phenomena and mechanisms. Corrosion Reviews, 2012, vol. 30 (3-4), pp. 105 - 123. DOI: 10.1515/corrrev-2012-0502.

11. Van den Eeckhout E., Laureys A., Van Ingelgem Y., Verbeken K. Hydrogen permeation through deformed and heat-treated Armco pure iron. Materials Science and Technology, 2017, vol. 33, iss. 13, pp. 1515-1523. DOI: 10.1080/02670836.2017.1342015.

12. Andronov D.Yu., Arseniev D.G., Polyanskiy A.M., Polyanskiy V.A., Yakovlev Yu.A. Application of multichannel diffusion model to analysis of hydrogen measurements in solid. International Journal of Hydrogen Energy, 2017, vol. 42, iss 1, pp. 699-710. DOI: 10.1016/j.ijhydene.2016.10.126.

13. Terent'ev V.F. Ustalostnaya prochnost' metallov $i$ splavov [Fatigue Strength of Metals and Alloys]. Moscow, Intermet Inzhiniring Publ., 2002, 288 p. (In Russian).

14. Lahdari A.A., Seddak A., Ovchinnikov I.I., Ovchinnikov I.G. Modeling of hydrogen embrittlement of a pipeline as a thin-walled cylindrical shell of a nonlinearly elastic material. Internet-zhurnal «NAUKOVEDENIE», 2017, vol. 9, no. 4. Available at: http://naukovedenie.ru/PDF/ 158TVN417.pdf

15. Ustalost $i$ vynoslivost metallov [Fatigue and Endurance of Metals, ed. by G.V. Uzhik]. Moscow, Izd-vo inostrannoy literatury Publ., 1963, 497 p. (In Russian).

16. Christmann K. Interaction of hydrogen with solid surfaces. Surface Science Reports, 1988, vol. 9, iss. 1-3, pp. 1-163. DOI: 10.1016/0167-5729(88)90009-X.

17. Mironov V.I., Emel'yanov I.G., Yakushev A.V., Lukashuk O.A. Development of rapid method for car steel quality control. Transport of the Ural, 2012, no. 2 (33), pp. 13-17. Available at: https://elibrary.ru/item.asp?id=17725311

18. Ovchinnikov I.I., Ovchinnikov I.G. Effect of hydrogen-containing environment at high temperature and pressure on the behavior of metals and structures. Internet-zhurnal «NAUKOVEDENIE», 2012, no. 4. Available at: https://naukovedenie.ru/PDF/60tvn412.pdf (accessed 20.09.2017).

19. Rebyakov Yu.N., Chemiavsky A.O., Chemiavsky O.F. Deformation and destruction of materials and structures in the diffusion. Vestnik YuUrGU, 2010, no. 10, pp. 4-16.

20. Lebedev A.A., Chausov N.G. Phenomenological fundamentals of the evaluation of crack resistance of materials on the basis of parameters of falling portions of strain diagrams. Problems of Strength, 1983, vol. 15, iss. 2, pp 155-160. DOI: 10.1007/BF01523460.

21. Smirnov S.V., Zamaraev L.M., Zamyatin A.N., Matafonov P.P. Short-term thermal cyclic creep and fracture of a VT1-0 titanium alloy in a hydrogen atmosphere. Russian Metallurgy (Metally), 2012, iss. 3, pp. 255-257. DOI: 10.1134/S0036029512030123. 
22. Smirnov S.V., Zamaraev L.M. Energy of activation of the VT5 and VT1-0 titanium alloys under short-term creep in air and argon. Diagnostics, Resource and Mechanics of materials and structures, 2016, iss. 6, pp. 100-110. DOI: 10.17804/2410-9908.2016.6.100-110. Available at: http://dream-journal.org/DREAM_Issue_6_2016_Smirnov_S.V._et_al._100_110.pdf

23. Velichko V.V., Mikheev G.M., Zabil'skij V.V., Maleev D.I. The influence of superlow concentration hydrogen on the mechanical properties of quenched 30KhG3A steel. FizikoKhimicheskaya Mekhanika Materialov, 1991, no. 1, pp. 112-114.

24. Kartashov A.M. Vliyanie vodorodnogo vozdeystviya pri vysokoy temperature $i$ davlenii na uprugie svoystva uglerodistoy stali [Effect of Hydrogen on the Elastic Properties of Carbon Steel at High Temperature and Pressure: Collection of Scientific Works of Graduate Students]. Leningrad, LITMO Publ., 1974, pp. 142-145. (In Russian).

25. Mironov V.I., Lukashuk O.A. Influence of Material Structural Inhomogeneity on Fracture Strength of Constructional Elements. Key Engineering Materials, 2017, vol. 735, pp. 89-112. DOI: $10.4028 /$ www.scientific.net/KEM.735.89. 
Подана в журнал: 29.11.2019

УДК 620.172.25

DOI: $10.17804 / 2410-9908.2020 .1 .024-033$

\title{
ВЛИЯНИЕ ТЕМПЕРАТУРЫ НАВОДОРОЖИВАНИЯ И РАСТЯГИВАЮЩЕГО НАПРЯЖЕНИЯ НА ПАРАМЕТРЫ ПОЛНОЙ ДИАГРАММЫ ДЕФОРМИРОВАНИЯ СТАЛИ О9Г2С
}

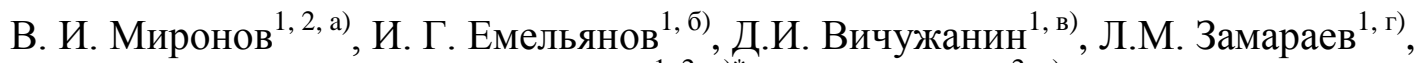
Д. А. Огорелков ${ }^{1,2, \text { д)* }}$, В. В. Яковлев ${ }^{2, \text { е) }}$

\author{
${ }^{1}$ Федеральное государственное бюджетное учреждение науки \\ Институт машиноведения Уральского отделения Российской академии наук, \\ ул. Комсомольская, 34, Екатеринбург, Российская Федераџия \\ ${ }^{2}$ Уральский Федеральный Университет, \\ ул. Мира, 19, Екатеринбург, Российская Федераџия
}

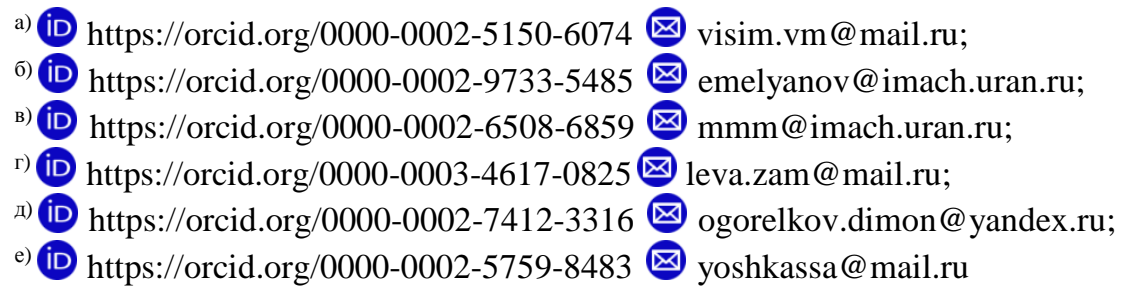

\footnotetext{
*Ответственный автор. Электронная почта: visim.vm@mail.ru, mailto: visim.vm@mail.ru Адрес для переписки: ул. Комсомольская, 34, 620040, Екатеринбург, Российская Федерация Тел.: +7-922-155-89-83
}

Концентрация водорода в материале образца зависит от его содержания в окружающей среде, внешнего давления, температуры, состояния поверхности и времени испытаний. Повышение температуры испытаний приводит к изменению фазового состава стали, активизации диффузионных процессов и к повышению содержания водорода. В результате снижаются предел прочности и предельная деформация, меняется угол наклона падающей ветви полной диаграммы деформирования (ПДД). По изменению параметров диаграммы наводороженного металла дается заключение об изменении его прочности, пластичности и трещиностойкости. Подход иллюстрируется результатами испытаний образцов из конструкционной стали 09Г2С, широко используемой в машиностроении. Установлено, что повышение температуры наводороживания снижает прочность и располагаемую пластичность стали, повышая трещиностойкость. Таким образом, экспериментально доказано, что параметры падающей ветви полной диаграммы чувствительны к концентрации водорода в стали. Отмечается качественная аналогия изменения механических свойств конструкционной стали от действия водорода и циклического нагружения. В этой связи делается заключение о принципиальной возможности исследования деградационных процессов разной природы на единой методологической основе. Представительными параметрами данных процессов могут служить параметры падающей ветви полной диаграммы деформирования.

Ключевые слова: водород, диффузия, эксперимент, полная диаграмма деформирования, температура.

\section{1. Введение}

Исследование закономерностей деградационных процессов в материале эксплуатируемой конструкции является основой физической теории надежности машин $[1,2]$. С точки 
зрения использования получаемых результатов в расчетах элементов конструкций несомненное преимущество имеет феноменологический подход. Кинетические уравнения, выраженные в терминах напряжений и деформаций, хорошо согласуются с уравнениями механики сплошных сред. Примером могут служить исследования циклической деградации ряда конструкционных сталей [3].

Общим характерным признаком процессов зарождения усталостной трещины и развития водородной хрупкости является переход изначально пластичного конструкционного материала в хрупкое состояние [4-10]. Кинетика обоих процессов в значительной мере определяется состоянием поверхностного слоя исследуемого объекта [11-15], а результаты лабораторных исследований сильно зависят от способа обработки образцов $[15,16]$. Приведенные аналогии послужили поводом для описания циклической и водородной деградации металлов с позиций авторского подхода, основанного на концепции полной диаграммы деформирования. Техника, средства и методика построения ПДД приведены в [3]. Простые испытания малых однократных образцов с построением полных диаграмм с падающей до нуля ветвью дают наиболее полное и логически завершенное представление о сопротивлении материала деформированию [3, 17]. Испытания таких образцов в жестком нагружающем устройстве позволяют оценить прочность, пластичность, трещиностойкость и энергоемкость материала по соответствующим параметрам ПДД.

Явление водородной деградации эксплуатационных свойств сталей, обнаруженное более 100 лет назад, в последние десятилетия привлекает особое внимание [14, 18-19]. В основном приводятся данные об изменении пределов прочности и текучести, относительного сужения при разрыве наводороженных образцов. Предполагается, что сравнение ПДД образцов в исходном и наводороженном состояниях даст более обстоятельную оценку изменения свойств материала от действия водорода. Цель работы - изучить влияние температуры наводороживания образца при неизменном давлении и времени выдержки в водороде на параметры статической полной диаграммы деформирования конструкционной стали.

\section{2. Средства и условия проведения экспериментов}

К настоящему времени преодолены технические трудности в построении полных диаграмм деформирования с падающей до нуля ветвью, отражающей стадию разупрочнения материала. Равновесное (без динамики) деформирование на стадии разупрочнения возможно при растяжении малых образцов [3] с размером рабочей части 2...3 мм в достаточно жесткой испытательной машине (рис. 1). Тонкостенный образец следует использовать при исследовании ичиклической деградации, поскольку усталостные повреждения концентрируются в поверхностном слое металла [3 и 13], а при наводороживании таких образцов сокращается время до насыщения.

Механические испытания образцов конструкционной стали 09Г2С с построением ПДД проводили на разрывной машине Instron 8801. Малая скорость деформирования 0,02 мм/мин и высокая жесткость машины позволяют строить падающую ветвь диаграммы без применения устройств повышенной жесткости. На рис. 2 в качестве примера приведены машинные диаграммы стали в координатах «растягивающее усилие $F$ удлинение $\Delta l \gg$ в исходном состоянии (1) и после отжига (2) при температуре $900{ }^{\circ} \mathrm{C}$ в течение трех часов. 


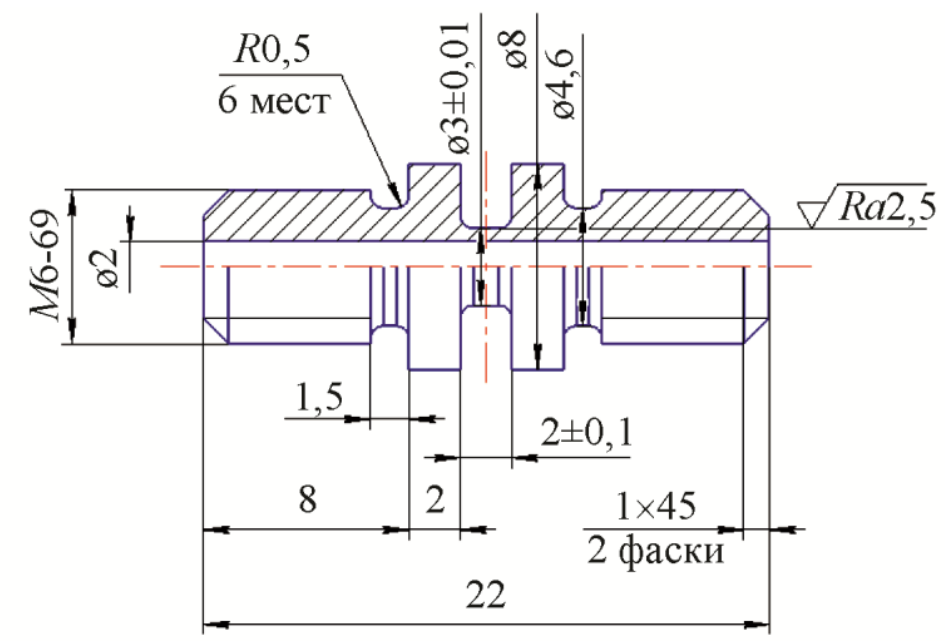

Рис. 1. Образец для испытаний с построением ПДД

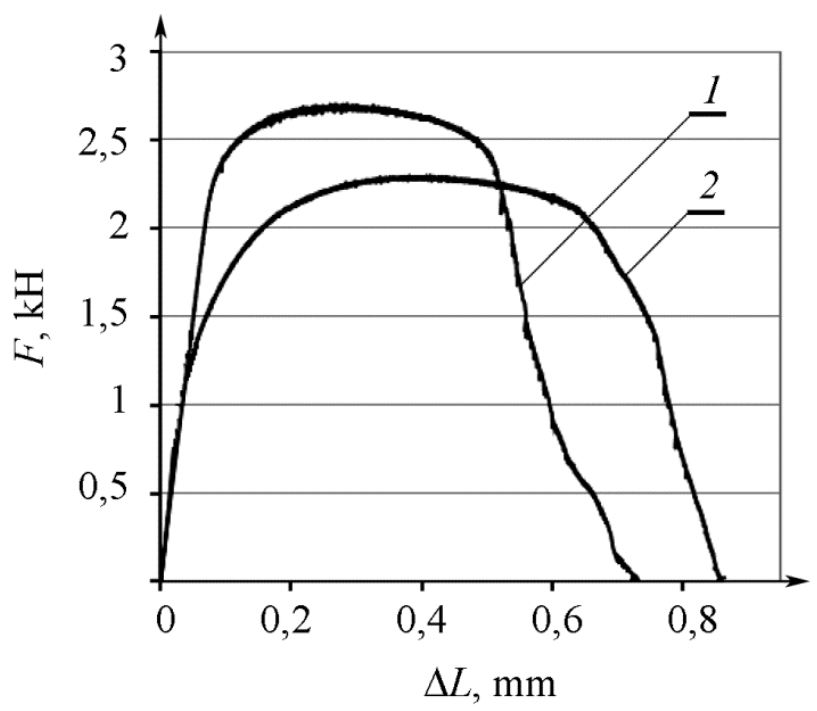

Рис. 2. Машинные диаграммы стали в исходном состоянии (1) и после отжига (2)

После отжига наблюдается значительное снижение максимальных деформирующих усилий и соответствующих удлинений. Возросла располагаемая пластичность, оцениваемая длиной диаграммы. Увеличился угол наклона заключительного участка диаграммы к отрицательному направлению оси абсцисс, что указывает на снижение трещиностойкости стали $[17,20]$. Практически не изменились энергоемкость материала, оцениваемая площадью под диаграммой, и модуль упругости. За пределом прочности понятие напряжения не вполне определено, поэтому машинная диаграмма не перестраивалась в условную, или какую-либо другую диаграмму. Тем не менее сравнение параметров машинной диаграммы позволяет судить об изменении механических свойств материала после отжига.

Наводороживание образцов проводилось на специализированном стенде с нагревом в среде чистого водорода без нагрузки и при фиксированных номинальных растягивающих напряжениях (рис. 3) [21]. 


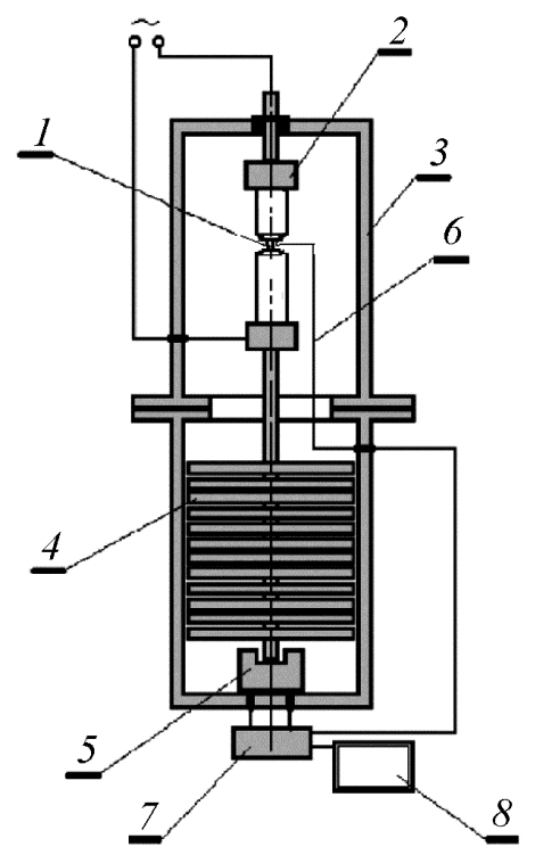

Рис. 3. Схема стенда

Для проведения испытаний образец (1) устанавливается в устройстве для подвески образца (2) и закрывают колпаком, образующим герметичную камеру (3). Растягивающее усилие создается набором свинцовых грузов (4). Образец нагревают путем пропускания переменного тока через силовой трансформатор с автоматизированным регулированием силы тока до 750 А. Температуру нагрева образца контролируют хромель-алюмелевой термопарой (6), привариваемой к каждому образцу в середине его рабочей части. Визуальное наблюдение за образцом в процессе испытаний осуществляется через кварцевые окна в колпаке. Удлинение образца фиксируется с помощью резистивного датчика (5). Датчик и термопара соединены с компьютером (8) через преобразователь сигнала (7). Воздух из рабочей камеры вытесняется газообразным аргоном из баллона с избыточным давлением 0,5 МПа. Система подачи водорода включает стандартный баллон и систему редуцирования и контроля. Давление водорода в камере 5 МПа поддерживают постоянным в процессе испытания. Стенд апробирован в испытаниях ряда материалов на кратковременную ползучесть в разных газообразных средах $[21,22]$. После наводороживания образцы снимали со стенда и испытывали при комнатной температуре на разрывной машине. При охлаждении металла и снижении давления большая часть поглощенного водорода выделяется. Учитывая высокую скорость процесса дегазации водорода, далее речь идет о влиянии остаточной концентрации водорода на диаграмму деформирования стали.

\section{3. Результаты исследований и обсуждение}

Известно, что растворение водорода в нержавеющей стали приводит к существенному разупрочнению [23], снижению пластичности и трещиностойкости $[4,19,23]$, модуля упругости [24]. Полное представление о свойствах стали одной марки можно составить по данным работ разных авторов. При этом используется материал разных плавок и разной термомеханической обработки, испытания проводятся по разным методикам и на образцах разных размеров и формы. Использование малых образцов позволяет провести масштабные испытания материала с одинаковой металлургической и технологической наследственностью (рис. 1). Так в сравнении машинных диаграмм стали 09Г2С выявлено существенное снижение прочностных свойств при отжиге (рис. 2). 
Влияние температуры предварительного наводороживания образцов в течение трех часов отражается в диаграммах на рис. 4, построенных при температуре $20{ }^{\circ} \mathrm{C}$. Диаграммы 1 на рис. 4 (те же, что на рис. 2) построены без предварительного наводороживания, диаграммы 2 для образцов, наводороженных при температуре $580{ }^{\circ} \mathrm{C}$, а диаграммы 3 - при температуре $900{ }^{\circ} \mathrm{C}$. Диаграмма 4 на рис. 46 получена после наводороживания при температуре $580{ }^{\circ} \mathrm{C}$ и растягивающем напряжении 10,5 МПа.
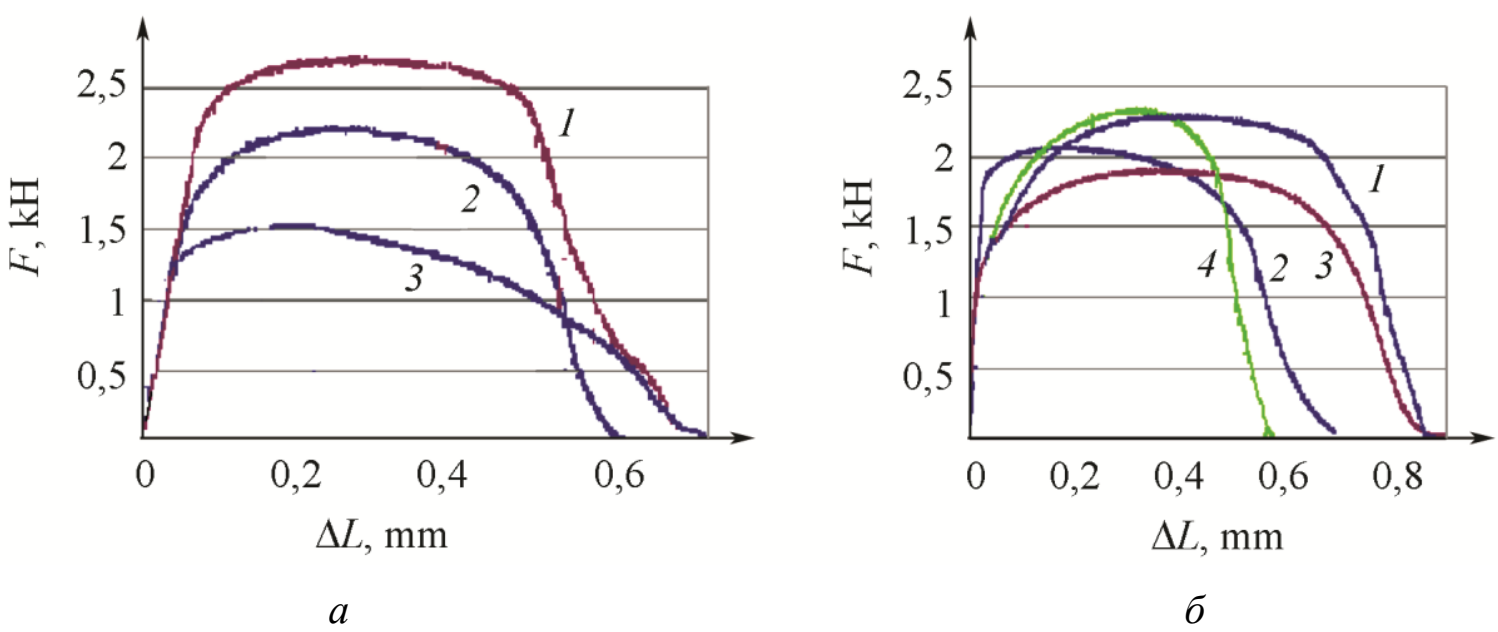

Рис. 4. Машинные диаграммы стали 09Г2С: $a-$ в состоянии поставки; $\sigma$ - после отжига при $900{ }^{\circ} \mathrm{C}$ в течение 2,5 ч

Сравнивая диаграммы 1,2 и 3 на рис.4 $a$ и рис.4 6 , можно заметить, что влияние температуры наводороживания на диаграмму материала в исходном состоянии и после отжига качественно одинаково. Максимальное усилие деформирования с ростом температуры наводороживания заметно снижается, особенно в состоянии поставки. Располагаемая пластичность, определяемая длиной ПДД, при температуре наводороживания $580{ }^{\circ} \mathrm{C}$ падает, но при температуре $900^{\circ} \mathrm{C}$ восстанавливается (кривые 3 на рис. 4). Последний результат не вполне совпадает с прямыми измерениями сужения сечения в шейке при разрыве. Так, для отожженных не наводороженных образцов значение относительного сужения $\psi=59 \%$ после наводороживания при температуре $580{ }^{\circ} \mathrm{C}$ снижается до $41 \%$, но при температуре $900{ }^{\circ} \mathrm{C}$ составляет всего $43 \%$.

Известно, что растягивающие механические напряжения ускоряют диффузионный процесс проникновения водорода в стали [19], особенно при невысоких температурах. Диаграмма 4, как и диаграмма 2 на рис. 4 б, построена после наводороживания при температуре $580{ }^{\circ} \mathrm{C}$. Но два груза в стенде создавали растягивающее напряжение 10,5 МПа (рис. 3).

Машинные диаграммы не приводились к инвариантному виду и значение максимума на диаграмме 4 следует считать случайным. Сравнивая диаграммы 2 и 4 , можно сделать качественное заключение: действие растягивающих напряжений проявляется в снижении располагаемой пластичности и трещиностойкости материала. Диаграмма растяжения стала короче и на падающей ветви, в диапазоне нагрузок $(1,4 \div 1,2)$ кН, появился круто наклонный участок, определяющий условия устойчивости деформирования и трещиностойкости $[20,25]$. При физическом обосновании параметра поврежденности необходимо учитывать снижение показателей прочности, пластичности и трещиностойкости материала. Показатели упругих свойств в данной работе не исследовались, но известно, что возможно снижение модуля упругости до 20 \% [19]. Для построения зависимостей изменения механических свойств с ростом концентрации водорода в образце необходимо дорогостоящее оборудование по ее определению. 


\section{4. Заключение}

Основной результат проведенных экспериментов заключается в том, что установлена чувствительность параметров ПДД к действию водорода. Испытания с построением полных диаграмм наводороженного материала дают комплексную оценку изменения его механических свойств с ростом температуры наводороживания. Наложение растягивающих напряжений ускоряет процесс перехода пластичной стали в хрупкое состояние, что проявляется в снижении располагаемой пластичности, определяемой длиной ПДД. Ранее показано, что параметры ПДД чувствительны к изменению химического состава, температуры испытаний, числа циклов тренировки. Таким образом, деградационные процессы разной природы и с разными механизмами реакции на внешние воздействия могут исследоваться в рамках метода полных диаграмм деформирования.

\section{Литература}

1. Болотин В. В. Ресурс машин и конструкций. - М. : Машиностроение, 1990. - 448 с.

2. Плютов Ю. А., Хомич Л. В. Новые подходы к анализу надежности транспортных машин // Фундаментальные исследования. - 2004. - № 6. - С. 70-72.

3. Емельянов И. Г., Миронов В. И. Долговечность оболочечных конструкций. - Екатеринбург : РИО УрО РАН, 2012. - 217 с.

4. Яковлева Т. Ю. Локальная пластическая деформация и усталость металлов. - Киев : Наукова Думка, 2003. - 238 с.

5. Мороз Л. С., Чечулин Б. Б. Водородная хрупкость металлов. - М. : Металлургия, 1967. $256 \mathrm{c}$.

6. Suzuki H., Fukushima H., Takai K. Role of Hydrides and Solute Hydrogen in Embrittlement of Pure Titanium // Journal of the Japan Institute of Metals. - 2015. - Vol. 79. - P. 82-88. DOI: $10.2320 /$ jinstmet.JC201402.

7. Tal-Gutelmacher E., Eliezer D. Hydrogen-Assisted Degradation of Titanium Based Alloys // Materials Transactions. - 2004. - Vol. 45, iss. 5. - P. 1594-1600. - DOI: 10.2320/matertrans.45.1594.

8. Further study of the hydrogen embrittlement of martensitic advanced high-strength steel in simulated auto service conditions / J. Venezuela, J. Blanch, A. Zulkiply, Q. Liu, Q. Zhou, M. Zhang, and A. Atrens // Corrosion Science. - 2018. - Vol. 135. - P. 120-135. DOI: $10.1016 /$ j.corsci.2018.02.037.

9. Garetta G., Cioffi P., Bruschi R. Engineering thoughts on Hydrogen Embrittlement // Procedia Structural Integrity. - 2018. - Vol. 9. - P. 250-256. - DOI: 10.1016/j.prostr.2018.06.038.

10. Lynch S. Hydrogen embrittlement phenomena and mechanisms // Corrosion Reviews. 2012. - Vol. 30 (3-4). - P. 105- 123. - DOI: 10.1515/corrrev-2012-0502.

11. Hydrogen permeation through deformed and heat-treated Armco pure iron / E. Van den Eeckhout, A. Laureys, Y. Van Ingelgem, K. Verbeken // Materials Science and Technology. - 2017. Vol. 33, iss. 13. - P. 1515-1523. - DOI: 10.1080/02670836.2017.1342015.

12. Application of multichannel diffusion model to analysis of hydrogen measurements in solid / D. Yu. Andronov, D. G. Arseniev, A. M. Polyanskiy, V. A. Polyanskiy, Yu. A. Yakovlev // International Journal of Hydrogen Energy. - 2017. - Vol. 42, iss 1. - P. 699-710. DOI: $10.1016 /$ j.ijhydene.2016.10.126.

13. Терентьев В. Ф. Усталостная прочность металлов и сплавов. - М. : Интермет Инжиниринг, 2002. -288 с.

14. Моделирование водородного охрупчивания трубопровода как тонкостенной цилиндрической оболочки из нелинейно упругого материала / А. А. Лахдари, Айсса Седдак, И. И. Овчинников, И. Г. Овчинников // Интернет-журнал «НАУКОВЕДЕНИЕ». - 2017. Том 9, № 4. - URL: http://naukovedenie.ru/PDF/58TVN417.pdf 
15. Усталость и выносливость металлов / под ред. Г. В. Ужика : сб. статей. - М. : Изд-во иностр. лит., 1963. - 497с.

16. Christmann K. Interaction of hydrogen with solid surfaces // Surface science reports. 1988. - Vol. 9, iss. 1-3. - P. 1-163. - DOI: 10.1016/0167-5729(88)90009-X.

17. Разработка экспресс-метода для контроля свойств вагонных сталей / В. И. Миронов, И. Г. Емельянов, А. В. Якушев, О. А. Лукашук. // Транспорт Урала. - 2012. - № 2. - С. 13-17. 18. Овчинников И. И., Овчинников И. Г. Влияние водородосодержащей среды при высоких температурах и давлениях на поведение металлов и конструкций из них // Интернетжурнал «НАУКОВЕДЕНИЕ». - 2012. - № 4. - URL: https://naukovedenie.ru/PDF/60tvn412.pdf (дата обращения: 20.09.2017).

19. Ребяков Ю. Н., Чернявский А. О., Чернявский О. Ф. Деформирование и разрушение материалов и конструкций в условиях диффузии // Вестник ЮУрГУ. - 2010. - № 10. C. 4-16.

20. Lebedev A. A., Chausov N. G. Phenomenological fundamentals of the evaluation of crack resistance of materials on the basis of parameters of falling portions of strain diagrams // Problems of Strength. - 1983. - Vol. 15, iss. 2. - P 155-160. - DOI: 10.1007/BF01523460.

21. Short-term thermal cyclic creep and fracture of a VT1-0 titanium alloy in a hydrogen atmosphere / S. V. Smirnov, L. M. Zamaraev, A. N. Zamyatin, P. P. Matafonov // Russian Metallurgy (Metally). - 2012. - Iss. 3. - P. 255-257. - DOI: 10.1134/S0036029512030123.

22. Smirnov S. V., Zamaraev L. M. Energy of activation of the VT5 and VT1-0 titanium alloys under short-term creep in air and argon // Diagnostics, Resource and Mechanics of materials and structures. - 2016. - Iss. 6. - P. 100-110. - DOI: 10.17804/2410-9908.2016.6.100-110. URL: http://dream-journal.org/DREAM_Issue_6_2016_Smirnov_S.V._et_al._100_110.pdf

23. Вляние сверхмалых концентраций воды на механические свойства закаленной стали 30ХГСА / В. В. Величко, Г. М. Михеев, В. В. Забельский, Д. И. Малеев // Физико-химическая механика материалов. - 1991. - № 1. - С. 112-114.

24. Карташов А. М. Влияние водородного воздействия при высокой температуре и давлении на упругие свойства углеродистой стали : сб. науч. трудов аспирантов. - Л. : ЛИТМО, 1974. - C. 142-145.

25. Mironov V. I., Lukashuk O. A. Influence of Material Structural Inhomogeneity on Fracture Strength of Constructional Elements // Key Engineering Materials. - 2017. - Vol. 735. - P. 89-112. DOI: $10.4028 / w w w . s c i e n t i f i c . n e t / K E M .735 .89$. 